\title{
Moisture Level Sensor Using Node-Red
}

\author{
Maradani Bhuvana Chandra, Korada Puneeth, Gaurav Dubey
}

UG Student, Department of Computer Science, Lovely Professional University, Jalandhar, India

\section{ABSTRACT}

\section{Article Info}

Volume 7, Issue 2

Page Number: 406-412

Publication Issue :

March-April-2021

\section{Article History}

Accepted : 15 April 2021

Published : 22 April 2021
This paper presents the implementation of an Internet of Things (IoT) application that performs the Moisture level sensing through node MCU and Raspberry $\mathrm{Pi}$, and data transfer to the Cloud of the Amazon Web Services or Raspberry Pi. The implementation is done using programming the Node MCU using Embedded C, Raspberry pi is Operated using Raspbian operating system and AWS EC2 Internet of Things platform based on the Node-RED tool installed on the Raspberry Pi and the AWS EC2. The Interconnection of data from the Node MCU to Raspberry Pi is done by MQTT Data Transfer Protocol and Mosquitto Protocol.

Keywords : Node-RED, MSL, MQTT, Mosquitto broker.

\section{INTRODUCTION}

The Internet of Things (IoT) idea approves interfacing actual things with ingrained electronics, software, sensors, and connectivity, therefore, providing data swapping with manufacturers, operators, and/or other connected devices. In 2013, the Global Standards Initiative on Internet of Things characterized IoT as "the worldwide IT foundation that gives progress administrations (physical and virtual) by systems administration of things, in light of existing and interchange data and similar growth in the turn of events" [1]. For this reason, the expression "thing" addresses "the object of the actual world (actual things), the data or the word (virtual things), which can be distinguished and can be incorporated into Communication networks".

The IoT permits things to be seen and distantly constrained by the current organization framework, empowering a more straightforward combination of the actual world and PC frameworks, bringing about expanded effectiveness, exactness, and financial advantages, while diminishing human mediation. By adding sensors to the IoT idea, an assortment of innovations is created, like brilliant homes, savvy urban areas, keen lattice, savvy transport, and virtual force plants. Everything can be remarkably perceived through an embedded PC system to a framework, is interoperable inside the current web framework. The expression "Web of Things" was proposed by Kevin Ashton in 1999 [1].is evaluated that by 2022 there will be in excess of 50 billion interconnected contraptions. The result of the increment in web customers, and accordingly the expansion in gadgets associated with the Internet is the appropriation of IPv6 (Internet Protocol rendition 6) on the grounds that every gadget needs the exceptional IP address to give web network. Because of the amazingly enormous measure of information (Big Information

Copyright: @ the author(s), publisher and licensee Technoscience Academy. This is an open-access article distributed under the terms of the Creative Commons Attribution Non-Commercial License, which permits unrestricted non-commercial use, 
idea), the sensors gather continuously, they are in most cases put away and prepared on a Cloud [3]. Their preparation gives us a considerably more itemized knowledge of how things around us work, where issues emerge, and where and how to respond. The IoT as of now changes our regular daily existence since numerous objects are furnished with smaller than expected recognizable proof gadgets or machine- coherent identifiers.

\section{LITERATURE SURVEY}

IoT refers to the a cluster of intelligent devices \& Systems connected with intelligence to gather data from embedded sensors, smart sensors and actuators and other physical objects. In the Future days, IoT will be expand rapidly, and a new dimension of services and utilisations that enhance the quality of consumers life and enterprise's productivity, unlocking an opportunity. Now this time Mobile networks already deliver connectivity to a wide range of devices, which can approve the development of new services and applications. This new wave of connectivity is far better than laptops and tablets, to connect cars and buildings, traffic control and smart meters with the anticipation of intelligently connecting almost anything and anyone.

IoT sensor integration to Node-RED platform $[\mathrm{x}]$ : This paper reviews the working of node-red development tools along with the history and development process of node-red like why Node-RED came into picture and what is the root cause of developing this tool. By this paper, we are trying to explain different functions of Node-red like continuous monitoring the flow and writing the reading data to the Json file. In this paper exploration to new Technologies which can merge to it like GPIO, Raspberry $\mathrm{Pi}$, etc. Also a good amount of explanation about components of Node-red light up like Node Panel, Flow Panel, Info and debug panel are explained in this paper. As data collection and storage come into the picture, now for storing large amounts of data collected by the moisture level sensors every second is difficult. So it is now feasible to store and collect at personal computers and hard disks or using cloud technology. Starting from explaining the cloud to working in a network like how the cloud can connect the server and ways in which how the cloud is helpful to industry. Software as a Services (SaaS), Platform as a Services (PaaS), and Infrastructure as a Service (IaaS) these platforms of services provided and constructed by the cloud to the users. At the same time, AWS EC2 platform also introduces the same types.

\section{Development Tool of Node-RED}

Basically, Node-RED is an open source and flowbased development tool which is used to integrate the IoT hardware devices, Application Programming Interfaces which are called as API's and online services developed by IBM Emerging Technology. Node-RED is built on Node.js platform which is a free JavaScript-based tool and provides a flow editor that is based on a visual browser ie., visual browserbased flow editor. Some appropriate icons are given to represent the nodes of the system. It can be operated in two ways: dragging, dropping, and connecting the nodes as wiring them up, or importing the JavaScript code. These nodes have different functions and do have different operations, such as the debug out node is used to monitor the flow, and Raspberry Pi node to read and write with Raspberry Pi GPIO pins. The flows which are created are stored using JavaScript Object Notation (JSON). In order to create flows for data processing, Node-RED enables developers in wiring up input, output and processing nodes, controlling things, and sending alerts . Node-RED basically works on the principle that enables the connection of web services and node customization 
and to do things, in performing some functions such as sending data which is collected by sensors via email and to services like Twitter, etc.,

Basic components of Node-RED:

1. Node Panel,

2. Flow Panel,

3. Info panel and Debug Panel.

Node-RED is a great tool to be used in creating prototypes. Node-RED allows instant creation of Applications, especially applications like IoT applications. This tool helps to enable engineers and technicians in creating and configuring the real time applications on end-devices in a simple and easy manner.

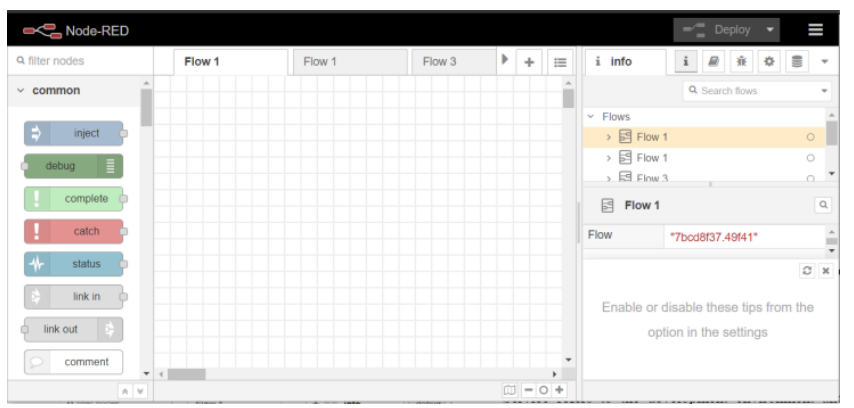

Fig 1 : The above shown refers the Standard Dashboard of Node-RED

\section{MQTT Protocol}

It is a machine -to - Machine IOT property Protocol. it had been designed as a particularly light weight publish/subscribe message transport. It establishes simple communication between multiple devices. it's a straightforward electronic communication protocol designed for affected devices with low information measure It is an ideal answer for net of things applications. MQTT permits to Send a command to regulate Associate in Nursing output Like Turning on lightweight and browse \& Publish knowledge the essential idea of MQTT is Publish/ Subscribe System,
Topics, and Brokers. a tool will Publish a message to the opposite devices wherever your device will subscribe a selected topic to receive those messages. If Device one Publishes on a subject and device a pair of Subscribes to identical topic within which device one is publication. So, device 2 receives the message. The messages are exchanged between the device nodes even it is a command or data. topics are the way to raise the interest for messages or specifies wherever the user need to publish topics are represented with strings separated by "/" slash indicates the level of the topics for example there is house which consists of bedroom with a light the topic is described as "house/bedroom/light". To switch on the light send a message to the topic using the Node-Red to switch on, any node can subscribe that is same topic therefore, it will receive on message and the light will turn on. Broker is primarily chargeable for receiving all the messages filtering the messages \& Publishes the messages to any or all signed The data in the form of messages to all the subscribed clients. There were $\mathrm{N}$ number of Brokers which we install on Raspberry pi.

\section{Moisture Sensing level}

Moving towards the next thing that is MSL (moisture sensor level) This is how this sensor is going to work with soil moisture sensor comprises two tests that measure the volume of water in the soil. The two tests permit the electric flow to go through the dirt and, as indicated by its obstruction, gauges the moisture level of the soil. When there is more water, the soil directs greater power, which implies that the opposition will be less. So, the moisture level will be higher. Dry soil lessens conductivity. Along these lines, when there is less water, the dirt directs less power, which implies it has more obstruction. So, the dampness level will be lower. Let's see how the sensor works in this scenario Moisture Sensor utilizes capacitance to gauge dielectric permittivity of the encompassing medium. In soil, dielectric permittivity 
is an element of the water content. The sensor makes a voltage relative to the dielectric permittivity, and thus the water substance of the soil. This much information gives a fair idea about how the sensor works.

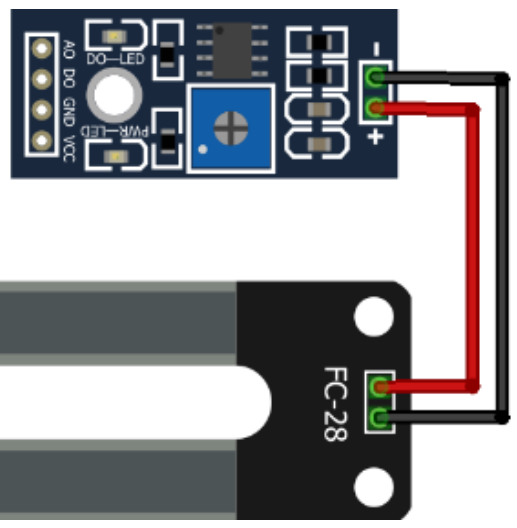

Fig 2 : The Above shown figure is the hardware structure of FC-28 Soil Moisture sensing Module

\section{CLOUD TECHNOLOGY}

Cloud technology represents the delivery of computing resources and storage capacity as a service for a heterogeneous

group of end users. The concept of Cloud technology relies on

sharing resources over the network, most often the Internet. Therefore, the Cloud in some way provides service delivery instead of the product itself. Endusers access Cloud applications through a web browser or mobile application on a mobile phone, while software and user data are located on servers in a remote location. The Cloud provides, at any time instance, the ability to access applications, data, data storage services, and does not require from the user to know the physical location of the service system. The Cloud is largely present in particular form, such as social networks, e-mail services or smart phones, etc. Moreover, the Cloud is a service that offers unlimited amounts of all resources (hard disk, processor, memory, etc.) when we really need it and to the extent that it suits our needs by doing everything we can to independently control it.

There are three types of Cloud technology:

- Software as a Service, SaaS,

- Platform as a Service, PaaS,

- Infrastructure as a Service, IaaS.

The basis of Cloud technology is a convergent infrastructure, made up of different IT technologies linked to one logical and functional entity, such as abstraction of physical resources by virtualization, as well as resource sharing. In the computing Cloud model, we distinguish two parts of the system: the front end, which is a user part and includes all the parts of the user-controlled infrastructure as well as the user's own way of accessing the service and the back end, which includes an infrastructure of Cloud providers.

\section{A Cloud platform (Platform as a Service)}

Service refers to the development environment and the required package of software subsystems. The user can develop, test, and distribute his own applications that run on the infrastructure of a Cloud service provider. Provider is in charge of a platform and executable environment, which most often include servers, network infrastructure, Datacentre, operating systems, and programming languages. The user has control over applications and the middle layer, while the Cloud service provider controls other layers of infrastructure, but the user can have the ability to choose the structure of the environment. In general, the team working on software development is not limited by the geographic location of resources or other team members. Examples of Cloud platforms are Amazon Elastic Beanstalk, IBM Bluemix, Google App Engine, and Microsoft Azure. 
Platform services can be used for applications or to implement a private Cloud environment. The platform provides capabilities such as easy and fast scaling and expanding resources as needed, adding new servers or services, high availability, and minimal downtime in the event of a failure some segment of the Cloud infrastructure itself or server, with the automatic migration and restart of the server on a healthy segment of the Cloud platform.

Cloud platform implies using the operating system via the Internet, without the need for download and installation. PaaS gives a variety of Cloud services that support and upgrade in all the parts of application development cycle, which consists of IDE's Version Control, Source Code Control, code tracking interactive tests for multiple users, and more. PaaS solutions are development platforms in which development tools are located on Cloud and accessed using the web browser.

\section{B. AWS EC2}

Amazon web services Elastic Compute Cloud is a integral service given by the Amazon though its AWS Clouds Services over the Cloud computing platform, the service gives the access to the user to create virtual Pc's or Laptops that are configurable with respective to lot many parameters like RAM, ROM, Graphic Card and many more. This EC2 Provides its applications named as a instance that means a virtual computer which is hosted over the internet which is allocated with a IP Address Probably of IPV4. Generally, user can Create Launch and terminate server instances as of his wish any time over the globe. it also provides with the classification with respective to geographical location whatever the user wish. This AWS EC2 was introduced in November month of 2010.

\section{IMPLEMENTATION}

For the Hardware Implementation the setup includes Raspberry pi, Node MCU(ESP8266), HC-28 Module, power Sources and some connecting Wires.

The Operating System Used for Raspberry $\mathrm{Pi}$ is Raspabian based on UNIX/INUX based Environment, in the Raspabian the Node red is Preinstalled, as a part of communication protocols Mosquitto broker must be installed over the Raspberry $\mathrm{Pi}$ even Mosquitto is also free ware software which is globally available.

\section{Commands used to install Mosquitto Broker :}

1. wget

http://repo.mosquitto.org/debian/mosquittorepo.gpg.key

To use the new repository you should first import the repository package signing key follow the below command, the wget command is used to download single file and stores in a current directory.

2. sudo apt-key add mosquitto-repo.gpg.key The above command is used to download Mosquitto Broker.

3. sudo apt-get update this command is used to update the packages over the Rpi.

4. apt-get install mosquitto-clients This command is used to install the mosquitto Clients.

5. mosquitto_sub-d-t armtronix_mqtt This Command is used to check the status of the Broker.

1. Setting up the Arduino IDE For Node MCU : Once the Arduino IDE is Installed over the Computer then need to add the ESP8266 Hardware Boards then the firmware of the board the complier maths equally that reflects the proper Connection. follow the next instructions to install the ESP8266 board in your Arduino IDE: 
2. In your Arduino IDE, go to File> Preferences.

3. Enter http://arduino.esp8266.com/stable/package_esp8 266com_index.json into the "Additional Boards Manager URLs" field as shown in the figure below. Then, click the "OK" button.

4. Open the Boards Manager. Go to Tools > Board > Boards Manager.

5. Search for ESP8266 and press install button for the 'ESP8266 by ESP8266 Community'.

6. That's all. It will be installed after a few seconds.

Node-RED runs from the terminal with command: nodered-start. Accessing over the browser by entering the IP address of Raspberry Pi and the 1880 port, a Node-RED interface will be displayed, confirming that the installation was successful. The appearance of the Node-RED editor is shown in Fig.1. Pressing CTRL + C stops the execution of the tool, and Node-RED is switched off by the command: nodered-stop.

\section{Circuit construction:}

FC-28 sensor has 4 pins A0, D0, VCC, GND where A0 \& D0 refers for data inputs and VCC, GND refers for voltage input.

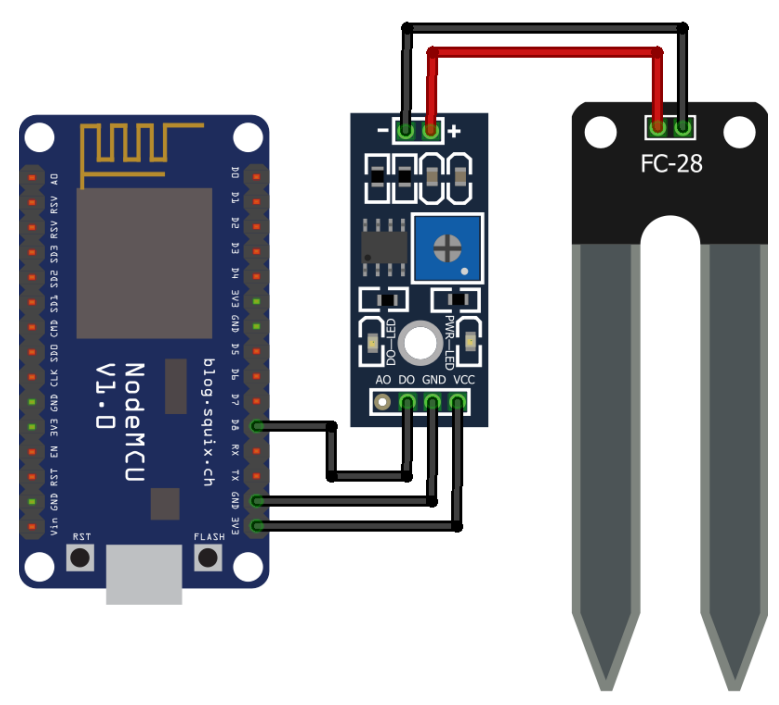

Fig 3 : The above shown Figure Display's the connection between Node MCU and FC-28 Module.
Where D0 is Connected to D8 of Node MCU.

Flow construction in Node-RED editor :

The Flow is constructed such a way that MQTT in takes the data from he broker and shares to node red as a string that goes to function block and then goes to the output gauge and graph.

The payload gives the data from the function block and shows over the debug dashboard located left side of the workspace.

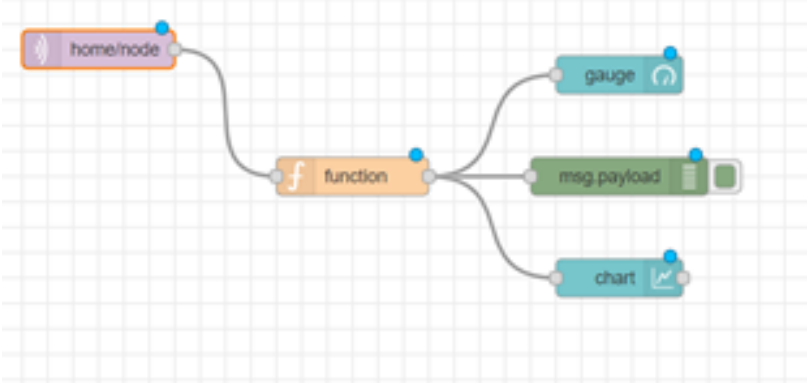

Fig 4 : The above figure shows the structural flow of node-red workspace. That the MQTT In node (home/node) push the MQTT Data from the mosquitto broker and pushes to Function Block checks the conditions of the string, then string data is given to payload that displays at Debug Section and the data is shared over the gauge and Chart for the better Visual Representation

The Figure6 shows 2particular outputs from the above Discussed Paper. The Right part shows output from the Debug Section which is generated by the intervene of msg. payload pallet. This is like a on system/ on dashboard output which deliberately shows the required or selected nodes. The Left side of Figure shows the graphical representation of data in 2 models i.e Chart and Gauge where Charts shows Timestamping in $\mathrm{X}$ axis and MSL value in $\mathrm{Y}$ axis. The gauge shows according to the conditions with different colours with respective to value shown there. 


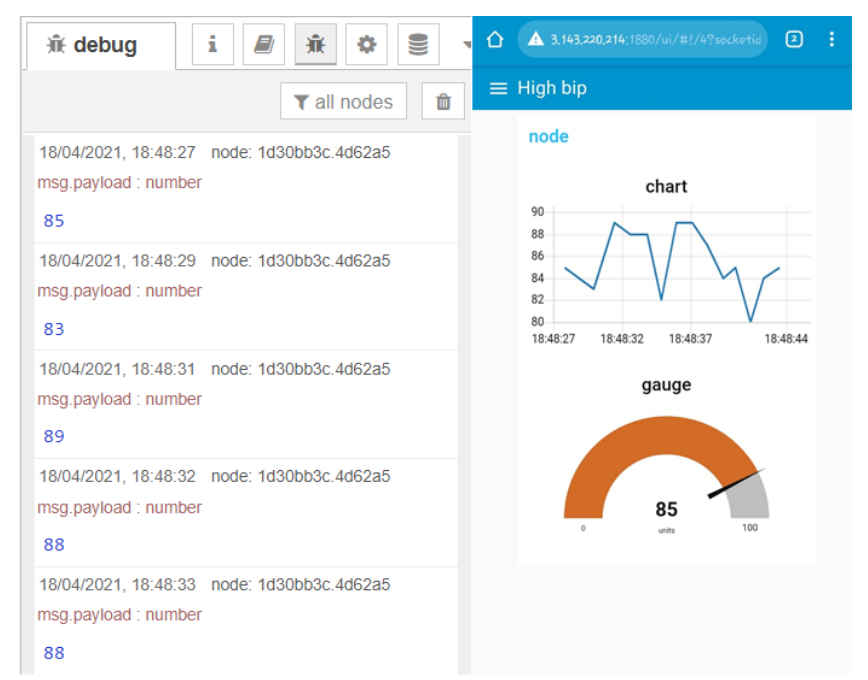

Fig : 5 Output Representation

\section{CONCLUSION}

The paper presents the implementation of an IoT application, created by using Raspberry Pi 3 model B, and a array of FC-28 Soil Moisture Sensor. The application on Rpi shows data collected from Raspberry Pi and FC-28 Soil Moisture Sensor. using Node-RED and WebSocket Protocol. With installed Node-RED and the flows which allow devices to send data and process commands can be built. Node-RED enables collecting data in the IoT systems and therefore provides a server function. Data on NodeRED are stored in internal data base. The collected data can be stored in data base created on some Data Base Server using nodes in Node-RED that enable it. Therefore, application retrieves data from that data base. Furthermore, collected data can be processed to obtain the desired information and to carry out statistics and analyses. Future work relates to creating and investigating various application scenarios using different sensors, in order to test different IoT concepts.

\section{REFERENCES}

[1]. Lekic, M., \& Gardasevic, G. (2018). IoT sensor integration to Node-RED platform. 2018 17th
International Symposium INFOTEH-JAHORINA (INFOTEH).

[2]. Rajalakshmi, A., \& Shahnasser, H. (2017). Internet of Things using Node-Red and alexa. 2017 17th International Symposium on Communications and Information Technologies (ISCIT).

[3]. Kodali, R. K., \& Anjum, A. (2018). IoT Based HOME AUTOMATION Using Node-RED. 2018 Second International Conference on Green Computing and Internet of Things (ICGCIoT)

[4]. Choopan Rattanpoka \& Somphop Chanthakit, An MQTT-based IoT Cloud Platform with Flow Design by Node-RED

[5]. John, J., Sarik, S., Baghini, M. S., \& Kasbekar, G. S. (2015). Design and implementation of a soil moisture wireless sensor network. 2015 Twenty First National Conference on Communications (NCC).

[6]. Kumar, M. S., Chandra, D. P., \& Manikandan, M. S. (2016). Monitoring moisture of soil using low cost homemade Soil moisture sensor and Arduino UNO. 2016 3rd International Conference on Advanced Computing and Communication Systems (ICACCS).

[7]. M.Ruggieri and H.Nikookar, "Internet of Things From Research and Innovation to Market Deployment".

[8]. E. Park, Y. Cho, J. Han, and S. J. Kwon, "Comprehensive Approaches to User Acceptance of Internet of Things in a Smart Home Environment," IEEE Internet of Things Journal, pp. 2342-50, 2017.

[9]. S. H. Shah and I. Yaqoob, "A survey: Internet of Things (IOT) technologies, applications and challenges," IEEE SEGE, pp. 381-5, 2016.

[10]. Node-RED guide, Web site: http://noderedguide.com

\section{Cite this article as :}

Maradani Bhuvana Chandra, Korada Puneeth, Gaurav Dubey, "Moisture Level Sensor Using NodeRed", International Journal of Scientific Research in Computer Science, Engineering and Information Technology (IJSRCSEIT), ISSN : 2456-3307, Volume 7 Issue 2, pp. 406-412, March-April 2021. Available at doi : https://doi.org/10.32628/CSEIT217283 Journal URL : https://ijsrcseit.com/CSEIT217283 